\title{
Tecnología y autogestión en cooperativas de trabajo*
}

\author{
Amalia Miano, David Burin y Ana Inés Heras
}

\section{Resumen}

¿Una forma de organización social diferente requiere de la implementación de tecnologías productivas y de organización diferentes? Partimos de esta pregunta para analizar el rol asignado a la tecnología en cooperativas de trabajo autogestionadas ubicadas en la provincia de Buenos Aires y Santa Fe. Nuestro análisis permite concluir que estas cooperativas, en tanto organizaciones sociales y productivas diferentes a aquellas que tienen al capital como eje organizador, a través de determinados mecanismos específicos -igualdad entre los miembros, posibilidad de tomar decisiones por parte de todos los trabajadores, orientaciones hacia la justicia, dispersión del poder-, posibilitan otras formas de interactuar e implementar tecnologías. Por otro lado, observamos que en aquellas cooperativas en las cuales los trabajadores asumían un fuerte compromiso político y cuestionamiento del orden social vigente, también había una preocupación por implementar modos de trabajo que

* Artículo recibido el 04 de abril de 2016. Aceptado 24 de agosto de 2016 
De Prácticas y discursos/ Universidad Nacional del Nordeste/ Centro de Estudios Sociales

resguarden la salud física y mental de los trabajadores. Estos modos se trasladaban también a las tecnologías que usan o generan estos grupos, de forma que las mismas pudiesen contemplar estos aspectos.

\section{Palabras clave}

Tecnología, Autogestión, Cooperativas de Trabajo.

\section{Abstract}

Does a different way of organizing require a different way of using technology? We start by asking this question in order to analyze what would technology role be in self-managed, workers' cooperatives in the Provinces of Buenos Aires and Santa Fe. We conclude that it is by way of complementary organizational mechanisms that constitute the core of workers' cooperatives that technology is re-conceptualized and used. These mechanisms are: equal member status, equal opportunity to make decisions, an orientation towards internal justice and a way of conceiving power as collective. On the other hand we have also documented that in cooperatives where members take up a strong political commitment and in turn, a strong critique over the hegemonic ways of power, that they implement ways to support healthy (both mental and physical) ways of doing their jobs. These healthy ways are also supported by some specific technological orientations.

\section{Keywords}

Technology- Self managed projects- Worker's cooperatives.

\section{Introducción}


En este trabajo abordaremos cuestiones ligadas al rol que se le asigna a la tecnología en cooperativas de trabajo autogestionadas de la provincia de Buenos Aires y de Santa Fe, Argentina. La mayor parte de los estudios sobre la tecnología estuvo dirigida a pensar críticamente el rol de la misma dentro del marco de la sociedad capitalista, comenzando ya por las reflexiones realizadas por Karl Marx (1980 [1968 ${ }^{1}$; 1984 [1847]; 1987 [1867]) y continuando luego con otros pensadores (Adorno y Horkheimer, 1969; Habermas, 1968; Marcuse, 2005; Mumford, 1982; Noble, 1984 y 2001). Así, se pueden encontrar debates en torno al determinismo tecnológico, a la alienación y descalificación de los saberes de la clase trabajadora al entrar en contacto con la maquinaria automática, consideraciones en torno a la escala o al tamaño de la tecnología, las formas de legitimar la dominación a través del dominio técnico racional, las consideraciones acerca de la dimensión política de la tecnología, entre otras aristas relevantes.

Más cercana a la problemática teórica planteada en este artículo existe una literatura generada en los países del campo socialista acerca de la organización del trabajo en cooperativas -por ejemplo, Adizes y Wolf, 1977-. También, en el campo del socialismo histórico ruso, existió una literatura apologética de la organización de la producción en manos del Estado socialista que pretendía ponderar las diferencias entre este tipo de organización del trabajo y la de los Estados capitalistas contemporáneos a su época. Se cuenta también con trabajos producidos por otros estudiosos del cooperativismo de trabajo agrario en varios lugares del mundo -USA, Israel, Rusia, Francia y México-, entre los cuales destacamos el trabajo de Henrik Infield, Sociología de la Cooperación (1971). Más cerca de nuestro momento histórico encontramos, a partir de 1980, a algunos estudios que aportan indicios para pensar el funcionamiento y las implicancias de la tecnología en contextos de organización que se rigen por parámetros diferentes a los

\footnotetext{
${ }^{1}$ Se trata de la obra Capital y tecnología (Manuscritos inéditos 1861-1863) publicada originalmente en 1968 por el Instituto de Historia de la Ciencia y de la Técnica de la Academia de Ciencias de la URSS para celebrar el $150^{\circ}$ aniversario del nacimiento de Marx. Contiene extractos de los cuadernos $\mathrm{V}$, XIX y XX de los manuscritos de Contribución a la crítica de la economía política, dedicados al análisis del plusvalor relativo, al desarrollo de las máquinas y al empleo de las fuerzas naturales y la ciencia en los procesos productivos.
} 
De Prácticas y discursos/ Universidad Nacional del Nordeste/ Centro de Estudios Sociales

del sistema capitalista de producción (Feenberg, 1991; Noble, 2001; Winner, 1986 y 1993).

A los fines de este trabajo, los grupos autogestionados con los cuales hemos desarrollado un proceso colaborativo de generación de conocimiento pueden caracterizarse como experiencias de organización económica alternativas, en términos de lo planteado por De Sousa Santos y Rodríguez (2012), ya que organizan la producción y la gestión del trabajo sobre la base de principios ligados a la justicia, tanto hacia el interior del propio grupo como en las relaciones que establecen hacia afuera -vínculos con proveedores, clientes, otras cooperativas, entre otros-. De esta manera, en estos colectivos hemos podido observar formas de producción, intercambio o consumo que buscan un equilibrio entre la sostenibilidad económica del proyecto productivo y formas basadas en la equidad y la mejora de las condiciones de vida de los integrantes de estos grupos, lo cual incluye formas de producir y relacionarse, de pensar el trabajo y realizarlo, y de establecer relaciones interpersonales e interinstitucionales. Además, hay un cuestionamiento y prácticas ligados a incidir de alguna manera en un contexto más amplio, que se materializa en la mayoría de los casos en demandas, protestas y propuestas realizadas al Estado (ver Heras y Burin, 2013; Heras, Miano y Burin, en prensa; Miano, 2013). A través de estas acciones, las organizaciones intentan sostener dos líneas constitutivas. Por un lado, desarrollar un emprendimiento productivo que sea económicamente sustentable y permita desplegar a sus integrantes otras actividades y concretar deseos y realizaciones personales -por ejemplo, a través del estudio, de la participación en actividades artísticas, deportivas o espirituales, de prácticas de mejoramiento de la salud o búsqueda de un equilibrio personal, etc-. Por otro, desarrollar prácticas identificadas con "lo político", en términos de los propios integrantes de las agrupaciones, trascendiendo así a su organización para articular con otras y producir una acción colectiva de alcance más amplio, tanto geográfico como institucional.

En tanto nuestro escrito se centra en el campo de los estudios sobre la tecnología, presentamos aquí cuestiones generales que hemos identificado al respecto de los debates en ese eje específico. Así, identificamos un debate polarizado entre el 
De Prácticas y discursos/ Universidad Nacional del Nordeste/ Centro de Estudios Sociales

determinismo tecnológico (Engels, 1972 [1874]; Heidegger, 2007 [1953]; Toffler, 1995) y el determinismo social (Bookchin, 1999; Mumford, 1982). El primero considera que el avance tecnológico tiene un desarrollo relativamente autónomo respecto al contexto socioeconómico y lo determina, en cambio el segundo reduce el desarrollo tecnológico a la dinámica de las fuerzas económicas y sociales. Al seguir esta lógica, el determinismo tecnológico conduciría a una inacción política ya que, si los dispositivos tecnológicos se desarrollan de forma autónoma a las relaciones sociales, los seres humanos nada podríamos hacer para reorientar el curso de ese desarrollo que en el sistema capitalista de producción está orientado hacia la maximización de las ganancias, la explotación de la naturaleza y los hombres, la alienación de los trabajadores, entre otros (Castoriadis, 2005). En cuanto al determinismo social, en términos generales, acordamos en que las relaciones sociales moldean la tecnología, sin embargo y siguiendo el planteo de Winner (1992), consideramos que no hay que perder de vista algunas cuestiones ligadas a la materialidad de la tecnología -que este autor presenta como "cuestiones técnicas"-, ya que en varias ocasiones esa materialidad es expresión de finalidades políticas. En función de esto, acordamos con Moles (1978) en que se da una sociodinámica en la cual las determinaciones se producen en ambos sentidos y donde, además de "la tecnología" y "las relaciones sociales", hay un tercer elemento como factor que dinamiza cambios en las otras dos esferas. Este tercer elemento sería el trabajador o bien, en el caso de las cooperativas, el propio colectivo. Es desde esta concepción que proponemos pensar en torno a quienes realizan investigación aplicada para desarrollar nuevas tecnologías, entendiendo aquí el término investigación aplicada en sentido lato: quienes identifican, analizan, interpretan y producen cambios tecnológicos en las organizaciones.

De esta manera, partimos de reconocer que el contexto social, la tecnología y los participantes directos de las organizaciones generan una sociodinámica en estas cooperativas autogestionadas, y reconocemos también que orientan sus propósitos de maneras diferentes a la producción capitalista. En consecuencia, nos preguntamos a los fines analíticos de esta comunicación: en estas formas de organización social alternativas, ¿qué fines explícitos persigue la incorporación de tecnología según los 
De Prácticas y discursos/ Universidad Nacional del Nordeste/ Centro de Estudios Sociales

perciben y expresan sus miembros? ¿Qué relaciones guarda dicha incorporación con los principios de autogestión y la orientación alternativa que estos grupos declaran sostener para su autogobierno y para organizar la producción? ¿Qué necesidades motivan la incorporación de tecnologías de gestión, organización, producción y gobierno?

Abordaremos estos interrogantes a partir del material generado con nueve cooperativas autogestionadas de la provincia de Buenos Aires y de Santa Fe. Tomaremos como referencia para el análisis algunas categorías provenientes del área de los Estudios Sociales de la Tecnología y la Filosofía de la Tecnología.

\section{Marco teórico}

\section{1. Caracterización de los grupos autogestionados}

En la misma línea de lo planteado por De Sousa Santos y Rodríguez (2012), en función de interpretar distintas experiencias de organización económica como alternativas, Aníbal Quijano (2012: 369) indica que si bien la idea y práctica política de una "existencia social sin explotación es, como se sabe antigua, su cristalización en la posición de que para realizarla es necesario un modo o un sistema alternativo de producción tiene apenas casi dos siglos, lo mismo que su referente, el concepto mismo de modo de producción. Ambos son, en ese sentido, productos específicos del moderno patrón del poder capitalista". Desde esta perspectiva, Quijano repasa las distintas iniciativas que fueron surgiendo a lo largo de la historia en los últimos doscientos años y muestra una variedad de situaciones que, a su vez, resultaron en propuestas situadas en contextos geopolíticos específicos. En este sentido, y coincidente con la literatura proveniente de la corriente denominada diverse economies (Gibson-Graham, 2008; Fickey y Hanrahan, 2014), el autor propone pensar que lo alternativo debe considerarse no solo como una respuesta ideológicamente organizada que conlleva un programa político sino, también, en el sentido de ser una respuesta a los contextos específicos de su surgimiento, de mostrar estrategias innovadoras de 
De Prácticas y discursos/ Universidad Nacional del Nordeste/ Centro de Estudios Sociales

sostenimiento, y -en todo caso- de contar con la capacidad de establecer redes con otros movimientos y situaciones.

Al tomar en cuenta estas cuestiones -y pensando en las formas alternativas de producción-, Quijano destaca para América Latina dos: la economía solidaria y la economía popular. Dentro de la economía solidaria la institución alternativa central es la cooperativa; en cuanto a la economía popular, las formas organizacionales son muy diversas y no necesariamente se formalizan en instituciones, ya que en general se trata de organizaciones pequeñas, donde priman las relaciones primarias y la lógica comunitaria. El autor destaca, a su vez, que en las relaciones de la economía solidaria, específicamente en las cooperativas de trabajo, existe siempre una posibilidad de desintegración o de falta de aplicación del sentido cooperativo y solidario que debería tener esta forma institucional, ya que pueden darse los casos donde bajo una formalidad cooperativa se recreen formas de relaciones productivas y sociales que tiendan a la explotación. Desde este punto de vista, las cooperativas de trabajo con que hemos producido el análisis presentado aquí tienen en común una posición reflexionada sobre estas cuestiones y es por ello que se autodenominan "cooperativas de trabajo autogestionadas", poniendo el énfasis en la cualidad de autogestión, que a su vez presupone una posición expresa a favor de la democracia interna, la justicia entre los asociados, y entre su proyecto y el entorno social. Hemos identificado así a cooperativas de trabajo que, según su propia caracterización, se orientan con un matiz explícito de autogestión. En tanto la Argentina continúa predominantemente organizando sus relaciones socioeconómicas en torno a los principios del capitalismo, estas cooperativas conviven con dicho marco. Asimismo, interpretamos que las mismas han buscado diferenciarse de otros grupos que fueron organizados con la forma de cooperativas, pero más bien a partir de una cierta regulación social expresa surgida del otorgamiento de planes sociales y de subsidios dirigidos a tales efectos, que en muchos casos produjeron en la Argentina de los últimos diez años un fenómeno específico de cooperativismo sin principios de autogestión $y$, a veces, sin generar tampoco un modo de producción cooperativo sostenible. 
De Prácticas y discursos/ Universidad Nacional del Nordeste/ Centro de Estudios Sociales

Partimos de suponer, junto a Lee (2006), que el valor suficiente para sostenerse en el tiempo en estas experiencias vincula, simultáneamente, prácticas materiales e ideas acerca de dichas prácticas que se traducen en formas concretas de llevar adelante un proyecto, en este caso, colectivo. Entre las cuestiones a las que atienden, indudablemente, surgen los medios de producción que, por definición, en estos proyectos son de propiedad colectiva.

\section{2. La cuestión específica de la tecnología y la autogestión}

En su libro El fectiche de la tecnología (2015), Novaes realiza un recorrido de diversos autores del campo de la economía solidaria (Gaiger, 2002; Singer, 1998; Tiriba, 1994) y concluye que, para éstos, la tecnología convencional puede ser usada sin significativas modificaciones en los emprendimientos autogestionarios. Apartándose de esta postura y explicitando una visión no neutral de la tecnología, el autor se propone el objetivo de relevar en empresas recuperadas -dos de Brasil y una de Argentina- en qué medida los cambios "del grupo social relevante que interviene en la fábrica" y "las posibilidades privilegiadas de intervención en el proceso de trabajo" (Novaes, 2015: 34) darían lugar a modificaciones en el plantel tecnológico de las empresas recuperadas.

Para nuestra investigación, hemos tomado el punto de partida y cuestionamientos que realiza Novaes. Sin embargo, como desarrollaremos más adelante, nuestro análisis abarcará tanto a empresas recuperadas por sus trabajadores como a grupos que desde sus inicios optaron por la autogestión como propuesta organizativa y también política. Así mismo, nos apoyamos en las formulaciones realizadas por Andrew Feenberg (1991), para quien el desarrollo tecnológico puede ser afectado por la acción del ser humano: es decir, este puede orientar el curso tecnológico a través de la lucha política. Puede haber, para este autor, al menos dos civilizaciones tecnológicas diferentes, basadas en distintas vías de desarrollo tecnológico. Una es la actual civilización industrial, en la cual existe una relación destructiva entre, por un lado, el industrialismo moderno y su tecnología de explotación y, por otro lado, la naturaleza y los seres humanos. La otra sería una civilización democrática en la cual los elementos subordinados del sistema técnico existente, es decir, los trabajadores que actualmente obedecen en la operación 
de los sistemas técnicos, tomarían el poder en la dirección que le imprimirían al desarrollo tecnológico. Las aristas implicadas en este proceso de transformación serían la democratización de la toma de decisiones sobre el diseño de las tecnologías -en tanto el diseño de las tecnologías se encuentra "plagado de consecuencias políticas" (Feenberg, 1991: 23)-, restituir "valores sociales" en esa instancia de diseño ${ }^{2}$-valores ligados a la libertad y lo que significa verdaderamente ser humanos- y "formas de control desde abajo", o bien, lo que nosotros llamamos autogestión: "ya que el centro del control técnico influencia el desarrollo tecnológico, nuevas formas de control desde abajo pueden fijar al desarrollo en un nuevo y original sendero" (Feenberg, 1991: 25). El autor plantea que estas dimensiones implicadas en el proceso de reforma de la tecnología solo pueden tener lugar en el contexto de una reorganización democrática. Si bien el escritor está haciendo una aseveración de nivel macro social, podemos pensar en trasladar estas dimensiones al análisis de experiencias de grupos autogestionados.

En una línea argumental similar a la de Feenberg, Winner plantea que las tecnologías encarnan formas específicas de poder, por lo tanto, una tarea fundamental para lograr una transformación es analizar sus implicancias políticas: "los sistemas socio-técnicos constan de regímenes con características que pueden ser descritas en términos políticos" (Winner, 1992: 294). Para el autor, como mencionamos más arriba, las posturas del determinismo social de la tecnología deben complementarse con un análisis que incorpore la materialidad de los artefactos técnicos, sus características y los sentidos de estos últimos, ya que en esas características puede estar encarnada la finalidad política de la tecnología. Es en este punto que el planteo de Winner se asemeja al de Feenberg, en tanto postula la condición de la participación de la ciudadanía en el proceso de desarrollo de la tecnología.

Esta democratización en el proceso de toma de decisiones acerca de una innovación tecnológica implica que: a) todos los grupos que van a resultar afectados deben

\footnotetext{
2 En cuanto a modificaciones en el diseño de la tecnología que incorporen "valores sociales", el autor da los siguientes ejemplos: "nosotros ya podemos sentir los grandes riesgos implícitos en la decisión técnica entre la producción por línea de montaje o por equipos de trabajo, entre diseñar computadoras para aumentar el control o para expandir la comunicación y entre construir ciudades en función de los automóviles o del transporte público" (Feenberg, 1991: 24).
} 
De Prácticas y discursos/ Universidad Nacional del Nordeste/ Centro de Estudios Sociales

participar dando sus opiniones en el estadio más temprano, es decir, cuando se va a definir qué será y a qué fines servirá esa tecnología; b) se debe formar políticamente a los ingenieros que van a diseñar las tecnologías ya que deben tener herramientas para evaluar las implicancias políticas que tendrán las tecnologías que desarrollan; c) la tecnología debe ser generada para tener un fin definido, es decir, se debe evaluar el para qué de esa tecnología y cómo se adecuan los fines elegidos a los medios de los que se dispone (Winner, 1992).

De acuerdo al historiador David Noble, hay una ideología profundamente arraigada en los seres humanos acerca de las bondades de la automatización y su vínculo con la idea de progreso. Esto implicaría una "visión darwiniana" de cómo evoluciona la tecnología en la cual, a través del paso del tiempo, se eliminarían las opciones que no servirían y sobrevivirían las posibilidades tecnológicas mejor adaptadas a los propósitos humanos (Noble, 2001). De allí la idea de que la última tecnología disponible en el mercado es siempre la mejor. Sin embargo, el autor da cuenta de que en realidad las tecnologías se encuentran modeladas por las relaciones de poder, ya que "ingenieros y científicos constantemente producen soluciones para aquellos que están en el poder (sus empleadores) y no pensando en los trabajadores" (p. 18). Por estas razones, para el autor, la tecnología encarna la visión del mundo de los que tienen el poder. En su obra La locura de la automatización (2001) también realiza un vínculo entre la automatización y el control de la clase trabajadora, sosteniendo que las máquinas automáticas subestiman los saberes de los trabajadores y eliminan cualquier posibilidad de intervención creativa.

Por último, para dar cuenta de los procesos de construcción, resignificación o adecuación de tecnologías, resultan relevantes los aportes del modelo constructivista de los estudios de la ciencia y la tecnología, que se propone investigar a través de casos empíricos los artefactos tecnológicos (Bijker, Hughes y Pinch, 1989). Estos estudios buscan potenciar la perspectiva que brinda poner el acento en la relación biunívoca entre tecnología y sociedad. De esta manera: "las sociedades están tecnológicamente configuradas, exactamente en el mismo momento y nivel en que las tecnologías son socialmente construidas y puestas en uso" (Thomas, Fressoli y Lalouf, 
De Prácticas y discursos/ Universidad Nacional del Nordeste/ Centro de Estudios Sociales

2008: 10). Esta amalgama de tecnología y sociedad es cristalizada en la metáfora de la seamless web -"red sin costuras"- entre naturaleza, cultura, sociedad y tecnología (Bijker, Hughes y Pinch, 1989; Latour, 2007). De esta manera, instituyendo a la tecnología en un nivel de análisis complejo: "lo socio-técnico", se superan los análisis deterministas en ambas vertientes -tecnológico y social- e instrumentalistas al abandonar los estudios de los condicionamientos y las consecuencias sociales del desarrollo y cambio tecnológico. En este contexto, son relevantes los aportes desarrollados desde lo que se conoce como Tecnologías para la Inclusión Social, orientadas a viabilizar el acceso igualitario a bienes y servicios al conjunto de la población a través de la participación de los potenciales usuarios en el proceso de diseño y tomas de decisiones para la implementación de estas tecnologías (Dagnino, Brandão y Novaes, 2004; Thomas, 2012).

Enmarcado en el enfoque constructivista de la tecnología y desde una aproximación etnográfica, contamos en nuestro país con estudios que analizan los ensambles sociotécnicos desplegados en una cooperativa de cartoneros (Carenzo, 2014 y 2015). Este estudio da cuenta de que los trabajadores de la cooperativa desarrollan una "profusa elaboración de taxonomías, procesos fisicoquímicos, artefactos y dispositivos materiales (maquinaria y herramientas)" (Carenzo, 2014: 115) para mejorar las labores cotidianas de los cartoneros y que esa elaboración está íntimamente ligada a la forma asociativa y resulta clave a su vez para modelar el proyecto político de la cooperativa en relación con el sector del reciclado.

A partir de estos desarrollos, podemos sistematizar algunas dimensiones teóricas relevantes para analizar los procesos de incorporación, adaptación o invención de tecnología en grupos de trabajo autogestionados. Ellas son:

- Los trabajadores de las cooperativas tienen la posibilidad de tomar decisiones sobre el proceso de trabajo, la incorporación de tecnología y los propósitos a los cuales debe servir esa incorporación.

- Es necesario prestar atención a los aspectos materiales de la tecnología, ya que en esa materialidad pueden estar implícitas determinadas finalidades políticas. 
- Los trabajadores de las cooperativas tienen la posibilidad de crear o generar otras maneras de hacer las cosas y también inventar tecnologías, dispositivos o procesos de trabajo.

En el próximo apartado expondremos las variables que aplicaremos para analizar el material generado durante el trabajo de campo. También daremos cuenta de nuestros vínculos y trayectoria previa de trabajo con grupos autogestionados y realizaremos una breve caracterización de estos últimos.

Posteriormente, vamos a referirnos a la cuestión que relaciona los medios de producción con el trabajo cooperativo autogestionado a través de la noción de tecnología que tomará en cuenta dos aspectos: el de la materialidad -equipamiento y herramientas- y el de la organización del trabajo. Los aprendizajes generados en estos dos aspectos se analizan en la presentación analítica de cada uno de los apartados, ya que hemos identificado aprendizajes referidos a los saberes específicos de los oficios y tareas que se requieren en cada cooperativa, a los aprendizajes ligados a la autogestión y a aquellos relacionados con la autonomía como proyecto político.

\section{Metodología}

\section{1. Forma en que se realizó el trabajo de campo: generación de datos}

Nuestro equipo de trabajo ${ }^{3}$ se encuentra indagando los vínculos entre aprendizajes, autogestión y autonomía desde el 2008. Desde ese año a la actualidad, se ha pasado por diversas etapas y financiamientos para esta línea de trabajo. Entre 2008 y 2011 identificamos organizaciones en diferentes regiones del país para relevar sus características en cuanto a la autoorganización. Entre 2011 y 2013 profundizamos el trabajo con algunos de los grupos que habíamos contactado en la primera etapa para comprender mejor sus prácticas (Heras, 2011 y 2015). Durante todo ese período se contó con financiamiento desde el sistema nacional de Ciencia y Técnica para llevar

\footnotetext{
${ }^{3}$ Este equipo cuenta con profesionales que tienen pertenencia en diversas instituciones y organismos de Ciencia y Técnica (IRICE-CONICET, UNSAM, Instituto para la Inclusión Social y el Desarrollo HumanoINCLUIR).
} 
adelante esta línea de trabajo ${ }^{4}$. En el 2012, y a partir de un trabajo colaborativo realizado de forma específica con ocho grupos autogestionados a través de un dispositivo que hemos denominado "Mesa Colectiva" (ver Heras y Miano, 2014; Mesa Colectiva, 2014), se presentó el proyecto Hacia nuevas formas de definir, crear e implementar tecnologías. Estudio etnográfico en proyectos de autogestión ${ }^{5}$. Este proyecto surge de relevar, tanto en la Mesa Colectiva como en el recorrido previo con los grupos, que la definición de tecnología y lo que se identificaría como tal en estos proyectos sería divergente a la forma en que se definen, diseñan e implementan tecnologías en emprendimientos que se rigen por la lógica capitalista de producción, orientados a maximizar la ganancia y basados en la explotación del trabajo. A su vez, en el marco de la Mesa Colectiva, pudimos observar que los grupos planteaban una necesidad de poder registrar las innovaciones de distinto tipo creadas en el seno de sus experiencias.

Como punto de partida hemos definido como proyectos autogestionados a colectivos de distinto tipo -cooperativas de trabajo y servicios, empresas recuperadas por sus trabajadores, movimientos sociales de obreros y trabajadores, asociaciones civiles, grupos de voluntarios autogestionados sin personería jurídica- que tienen en sus manos la gestión ya sea de sus medios de producción como de sus normas y decisiones. Dentro de estos colectivos, hemos elegido para trabajar específicamente a aquellos que además de conformarse como un proyecto autogestionado, asumen los principios del proyecto de autonomía (Castoriadis, 1990), que se caracterizan por permitir a sus miembros la participación directa en la construcción y aplicación de acuerdos y normas, por abrir espacios de interrogación sobre los sentidos construidos, por entender la búsqueda de la verdad como la posibilidad de examinar perspectivas diferentes y considerar como valores fundamentales la equidad, la justicia y la libertad (Heras, 2009 y 2011). En oposición podemos identificar a la heteronomía como el modo de pensar y organizar la sociedad que tiende a la jerarquía, a la toma de decisiones por

\footnotetext{
${ }^{4}$ Nos referimos a los proyectos PICT 0696 Aprendizaje y creación en proyectos de autonomía y PIP 0087 Aprendizaje y percepción de la diferencia en proyectos de autonomía, dirigidos ambos por la Dra. Ana Inés Heras. Asimismo, el proyecto llevado adelante por el Instituto para la Inclusión Social y el Desarrollo Humano con financiamiento del MCyT, Res. 460/13.

${ }^{5}$ PICT 1414, dirigido por Amalia Miano.
} 
De Prácticas y discursos/ Universidad Nacional del Nordeste/ Centro de Estudios Sociales

delegación, a la forma de pensar que le otorga una autoridad incuestionable a lo que está establecido sin animarse a desnaturalizarlo y cuestionarlo, y a la clausura de sentidos (Heras, 2011).

Para relevar las características específicas de las tecnologías en los grupos de trabajo autogestionados nos contactamos con nueve cooperativas de trabajo. Dos de ellas se encuentran en la provincia de Buenos Aires y las restantes, en la ciudad de Rosario, provincia de Santa Fe. Tanto las dos cooperativas de Buenos Aires como una de las de Rosario son empresas recuperadas por sus trabajadores, mientras que las restantes se han conformado desde sus inicios como cooperativas de trabajo. Estos puntos de partida diferentes son relevantes para el análisis ya que "no es lo mismo una fábrica recuperada que parte de una estructura jerárquica y vínculos previos con el sindicato del sector y donde los trabajadores llegan a la instancia cooperativa como la mejor alternativa para no perder el trabajo, que un colectivo autoconvocado que elige la autogestión por convicción ideológica" (Heras y Burin, 2014).

Con una de las cooperativas de la provincia de Buenos Aires hemos desarrollado un trabajo de campo etnográfico desde julio de 2012 hasta noviembre de 2013, con el fin de analizar los vínculos entre las tecnologías presentes en la planta y la forma de organización autogestionada. Se trata en este caso de una fábrica recuperada por sus trabajadores, ubicada en la localidad de Pilar, que actualmente cuenta con 30 socios que tienen una antigüedad promedio de entre treinta y cuarenta años de trabajo en la planta.

A partir del 2014, nos hemos contactado con las demás cooperativas de trabajo a las cuales hemos realizado entrevistas en profundidad. Específicamente con las cooperativas de la ciudad de Rosario, se ha establecido una modalidad de trabajo alrededor del cine debate y talleres de formación, debate conjunto y sistematización de las prácticas, siguiendo en este caso la metodología de trabajo ya probada en la Mesa Colectiva de Trabajo antes mencionada ${ }^{6}$. También se desarrollaron jornadas de observación participante en algunas de estas cooperativas con registro en video y con

\footnotetext{
${ }^{6}$ Todos los grupos de la ciudad de Rosario pertenecen al Movimiento de Trabajadores Autogestionados (MTA) y han sido ellos quienes han contactado a nuestro equipo para realizar un ciclo de cine debate.
} 
De Prácticas y discursos/ Universidad Nacional del Nordeste/ Centro de Estudios Sociales

posterior sistematización en notas de campo etnográficas, compartidos ambos registros luego con los trabajadores.

Todas las cooperativas contactadas pertenecen a distintos rubros productivos: un lavadero de ropa industrial, una fábrica de auto partes, una textil, dos almacenes naturistas, un delivery y restaurante de comida vegetariana, un emprendimiento que produce cerveza artesanal, un emprendimiento que produce medallones de legumbres, un bar y un centro cultural'.

\section{II.2. Categorías analíticas y construcción de matriz de variables para el análisis: generación del marco analítico.}

A continuación, presentamos las categorías de análisis y variables que construimos a partir de nuestro trabajo de observación y sistematización del material generado junto a las cooperativas para analizar, en el próximo apartado, los cruces entre estas categorías y las experiencias con las que trabajamos.

\section{1- Tecnologías de producción}

1-a. Compra de equipamiento nuevo o usado.

1-b. Reparación o adaptación de equipamiento disponible -por ejemplo, cambios ergonómicos en el diseño para comodidad y salubridad de los trabajadores o para facilitar el trabajo en equipo-.

1-c. Invención de equipamiento.

\section{2- Tecnologías de organización del trabajo}

2-a. Modificaciones en la línea de producción: cambios en la concatenación de la secuencia de pasos en el proceso de producción; ubicación relativa y relación entre las máquinas, y entre los trabajadores y las máquinas; reducción de tiempos de producción y en el movimiento de materiales a partir de modificar la cantidad de trabajadores que

\footnotetext{
7 Posteriormente y también a demanda de las cooperativas del MTA, se coordinó un taller sobre el contenido de los Reglamentos en las cooperativas de trabajo y los conflictos que pueden preverse y enmarcarse dentro de estos Reglamentos, y se formuló un proyecto de investigación para relevar la situación de 40 cooperativas rosarinas en cuanto a las exigencias formales, fiscales, legales, contables y previsionales que exigen los organismos de control estatal. Como mencionamos más arriba, de las cooperativas que pertenecen a este Movimiento, solo una es una empresa recuperada por sus trabajadores. En todos los demás casos, se trata de grupos que tienen en su mayoría entre cinco a siete integrantes que optan por conformar una cooperativa como una forma de trabajo diferente.
} 
opera en cada paso del proceso, integración de tareas de ejecución y concepción en un mismo trabajador; movilidad entre puestos de trabajo; cambios realizados en los productos; simplificación o eliminación de piezas innecesarias para facilitar la producción.

2-b. Generación de sistemas informatizados para la gestión de la producción o ventas.

2-c. Generación de sistemas de registro de procesos, fórmulas y/o recetas para determinados productos o ventas.

2-d. Espacios para la toma de decisiones sobre el proceso de trabajo -asambleas, reuniones, comisiones de trabajo-.

2-e. Generación de áreas o comisiones de trabajo.

\section{Resultados}

En un primer paso expondremos los cruces que realizamos entre las categorías de análisis y sus variables con cada una de las experiencias. Por una razón de espacio, no explicaremos dentro de la tabla especificidades de cada una de las tecnologías, cuestión que sí desarrollaremos en un segundo paso, en el cual daremos cuenta de desarrollos e implementación de tecnologías en las cooperativas. En este análisis abordaremos también las finalidades que persiguieron tales desarrollos y las dimensiones centrales que pueden desprenderse de las características específicas de la tecnología en estas cooperativas autogestionadas.

Categoría 1- Tecnologías de producción por unidad productiva (cooperativas) en el lapso (2012-2015).

\begin{tabular}{|c|c|c|c|}
\hline \multirow[t]{2}{*}{ Cooperativas } & \multicolumn{3}{|c|}{ 1- Tecnologías de producción } \\
\hline & $\begin{array}{l}\text { 1.a. Compra de } \\
\text { equipamiento nuevo } \\
\text { o usado }\end{array}$ & $\begin{array}{l}\text { 1.b. Reparación o } \\
\text { adaptación de } \\
\text { equipamiento } \\
\text { disponible }\end{array}$ & $\begin{array}{l}\text { 1.c. Invención de } \\
\text { equipamiento }\end{array}$ \\
\hline PRANA & SI & SI & SI \\
\hline PRONOAR & SI & NO & $\mathrm{NO}$ \\
\hline
\end{tabular}


De Prácticas y discursos/ Universidad Nacional del Nordeste/ Centro de Estudios Sociales

\begin{tabular}{|l|c|c|c|}
\hline Movimiento & SI & NO & NO \\
Cajonardi & SI & NO & NO \\
\hline Pichangú & SI datos \\
\hline Sattva & SI & SI & SI datos \\
\hline Inlackech & NO & SI & SI \\
\hline La Nueva Avan* & SI & Sin datos & Sin datos \\
\hline La Unión* & NO & & SI \\
\hline Textil Pigué* & & & SI \\
\hline
\end{tabular}

En el caso de la compra de equipamiento nuevo o usado, para las cooperativas que no son empresas recuperadas, el adquirir equipamiento es una condición para poder realizar las actividades productivas, de allí que todos estos grupos hayan tenido que realizar una inversión inicial para esa adquisición y en muchos casos, inversiones posteriores a medida que se han consolidado o debieron aumentar la producción u organizar mejor el espacio de ventas al ampliar los stocks de mercadería en el caso de los comercios. En las recuperadas vemos que en solo una de las tres relevadas se ha adquirido equipamiento. Las formas para lograr la compra de equipamiento son diversas: resignar un porcentaje de los retiros de los trabajadores por un período de tiempo, conseguir financiamiento a través de programas estatales o gestionar un crédito -tanto como cooperativa, como varios microcréditos individuales que se vuelcan a un fondo común-, ayuda financiera de parientes o amigos, entre otras. A su vez, la mayoría de las cooperativas accede a internet para investigar qué tipo de máquinas son las que les convienen, cómo funcionan y qué tipo de mantenimiento requieren. En cuanto a los conocimientos requeridos para utilizarlas, mencionan que en general no son máquinas que generen dificultades, por el contrario "tienen dos o tres botones nomás".

La incorporación de cada nuevo equipamiento es una decisión razonada en el colectivo y sometida a reflexión. Hemos observado que esta incorporación está supeditada a una evaluación sobre las metas productivas que se quieren alcanzar. Un término utilizado por dos de las cooperativas recuperadas para referirse al plantel actual de maquinarias es el de adecuación, es decir, para el nivel productivo actual, las máquinas con las que

\footnotetext{
${ }^{8}$ Se asigna un asterisco a las tres cooperativas que son empresas recuperadas.
} 
De Prácticas y discursos/ Universidad Nacional del Nordeste/ Centro de Estudios Sociales

cuentan son suficientes y adecuadas, en caso de aumentar el nivel de producción, sí tendrían que adquirir más máquinas. Esto nos lleva a poner en debate la idea de que para los trabajadores la última tecnología disponible en el mercado es la mejor. En realidad, el criterio no sería la novedad, sino si esa máquina podría adecuarse a los fines o metas que la cooperativa quiere alcanzar.

Por otro lado, observamos también que hay una valorización de la tecnología disponible que, interpretamos, puede atribuirse a varias razones. Por un lado, en el caso de las cooperativas recuperadas, algunos trabajadores hace alrededor de 25 años que están trabajando con las mismas máquinas. Esta experiencia de trabajo lleva a que se dé una especie de acoplamiento entre los tiempos de la máquina y del trabajador, un conocimiento muy profundo de la forma de operarla. Por ejemplo:

Claudio me mostró unos rodillos con los cuales hacen las rótulas, son rodillos que están muy gastados, me explicó. Me dijo que la muy buena calidad que tienen las piezas que ellos hacen se debe a la experiencia que tienen los trabajadores de la cooperativa, ya que las máquinas son muy viejas. El laminado de piezas, en cualquier fábrica de autopartes, se hace de forma automática, en cambio, con la máquina que tienen ellos, se hace de forma manual. Al ser el rotulado así, hace que la pieza sea de mayor calidad que las que están en el mercado. Sin embargo, el proceso es mucho más lento que con una máquina automatizada. Esta máquina manual "comprime" la pieza, la otra le quita pedazos y eso hace que baje la calidad. Con esta máquina manual el trabajador se da cuenta de que el rotulado ya está hecho por un sonido que emite la pieza, el trabajador tiene incorporado ese tiempo y sonido para retirar la pieza a tiempo. (Cuaderno de campo, cooperativa La Nueva Avan, 22 de octubre de 2012).

La valorización de la tecnología disponible, por otro lado, también se asocia con el trabajo artesanal. En varias ocasiones se destaca que al no ser maquinaria automatizada, esto permite que el trabajador pueda intervenir directamente en la materia y estas operaciones ligadas a "lo manual" dan lugar, por un lado, a que el trabajador pueda desplegar sus saberes $y$, por otro, a que las piezas o productos sean 
De Prácticas y discursos/ Universidad Nacional del Nordeste/ Centro de Estudios Sociales

de mayor calidad que los producidos por máquinas automáticas, según la percepción de los trabajadores:

Por ahí, por las condiciones en las que uno está, se tiene que dar mucha maña para sacar procesos nuevos y por ahí las máquinas que se necesitan para este tipo de proceso valen una fortuna, entonces se le busca una alternativa, una variante en que si bien el producto final no es igual, pero se asemeja lo más parecido posible a lo que nos están pidiendo, se va innovando y se van haciendo cosas. Generalmente como este tipo de trabajo es muy artesanal, entonces por ahí con viejas técnicas que hemos aprendido en el lavadero en que hemos trabajado en relación de dependencia, entonces se implementan esas técnicas que son artesanales, pero que te llevan mayor tiempo que teniendo una máquina (...) Hoy tenemos clientes que han dejado de llevar a otros lavaderos que tienen mayor tecnología por la calidad también que se le brinda acá al producto, por ahí con menos tecnología. (Trabajador de cooperativa La Unión, 26 de julio de 2014).

Reconocemos también que, si estamos evaluando las posibilidades de que la tecnología pueda orientarse a alivianar el trabajo humano, contar con equipamiento automatizado sería una buena opción para los trabajadores de las cooperativas. De hecho, en todos los grupos en los cuales se ha incorporado equipamiento, los trabajadores reconocen la agilidad y mejoramiento en las condiciones de trabajo -por ejemplo, la compra de una amasadora y una sobadora en el caso de la cooperativa que hace los medallones de legumbres, ollas de acero inoxidable que dejan levar los granos a una temperatura estable en el caso de la fábrica de cerveza, secadoras automáticas en el lavadero industrial-. Consideramos que en todo caso, la evaluación a hacerse no es si la automatización en sí misma es conveniente o no, sino, en el caso de estas cooperativas, qué se hace con el tiempo que queda liberado a partir de la incorporación de maquinaria automática.

Mientras que en una empresa capitalista ese tiempo se traduce en plusvalor para el dueño de la fábrica -que además puede estar asociado a la eliminación de puestos de trabajo-, la característica específica de estos grupos es que ese tiempo se utiliza para acortar las horas de la jornada de trabajo, poder hacer más recreos, realizar reuniones o 
De Prácticas y discursos/ Universidad Nacional del Nordeste/ Centro de Estudios Sociales

asambleas, desayunar y almorzar juntos, y reunirse nuevamente a charlar al final de la jornada de trabajo, hacer pruebas orientadas a diversificar los productos que se realizan, o bien también aportar trabajo a la comunidad. Esto nos da la pauta de que en estos grupos pareciera buscarse un equilibrio entre un nivel de productividad que permita sostener económicamente al proyecto y un ritmo de trabajo con cierto bienestar que no genere condiciones de alienación.

En cuanto a los procesos de adaptación o invención de tecnología, hemos observado que la mayor parte de ellos se realiza para mejorar las condiciones de trabajo modificar la altura de las hornallas para que el calor no vaya directamente al vientre de los trabajadores, soldar rueditas a una estructura de metal para poder hacerla circular con menor esfuerzo, cambiar el material de un cerramiento para que ingrese más luz al ambiente, construir extractores de aire con las aspas de ventiladores para extraer las partículas de polvo suspendidas en los ambientes de trabajo, entre otros- y para simplificar la producción -"muñecos" para poder trabajar sobre camisas, sistemas de secado de medallones de legumbres utilizando planchas de metal ranurado y esmaltado que fue material de descarte de una fábrica, soldadura de moldes individuales en una estructura más grande para realizar cortes múltiples de las masas-. Una de las cooperativas cuenta incluso con un cuaderno llamado Desarrollos tecnológicos, donde van anotando ideas para generar tecnologías.

Así mismo, identificamos en esta categoría cuestiones ligadas a la forma de aprender. En cuanto a los saberes específicos del oficio, las técnicas y el proceso de trabajo, los trabajadores mencionan a la práctica y la interacción como dos elementos imprescindibles para adquirir aprendizajes. De allí que, cuando ingresa un nuevo compañero al proyecto, tiene, en la mayoría de los grupos, un primer momento en que verá cómo otro trabajador más experimentado realiza las tareas para, en un segundo momento, poder realizar esas tareas por sí mismo. De este modo, interpretamos que existe un pensamiento acerca del proceso productivo más bien ligado a espacios de interacción cara a cara que se construyen específicamente para ir guiando a quien se inicia y también para ir propiciando una objetivación de la práctica productiva en términos de trabajo colectivo. 
En los proyectos relevados, además, existe una pregunta siempre presente acerca del aprendizaje vinculado a la producción, tanto en lo que refiere a los saberes ligados a la producción específica -sea de alimentos, repuestos, producción textil- como a los conocimientos vinculados a la producción cooperativa. En nuestra observación del trabajo y en entrevistas se identifican aspectos ligados igualmente a la formación sobre fijación de precios, aspectos contables y legales, o realización de reglamentos acordes al enfoque del cooperativismo de trabajo.

Categoría 2. Tecnologías de organización del trabajo por unidad productiva (cooperativa) en el lapso (2012-2015).

\begin{tabular}{|c|c|c|c|c|c|}
\hline \multirow[t]{2}{*}{ Cooperativas } & \multicolumn{5}{|c|}{ 2- Tecnologías de organización } \\
\hline & $\begin{array}{l}\text { 2.a. } \\
\text { Modificaciones } \\
\text { en la línea de } \\
\text { producción o } \\
\text { en las ventas }\end{array}$ & $\begin{array}{l}\text { 2.b. Generación de } \\
\text { sistemas } \\
\text { informatizados para } \\
\text { la gestión de la } \\
\text { producción o la } \\
\text { venta }\end{array}$ & $\begin{array}{l}\text { 2.c. Sistemas de } \\
\text { registro de } \\
\text { procesos, } \\
\text { fórmulas, } \\
\text { recetas }\end{array}$ & $\begin{array}{l}\text { 2.d. Espacios } \\
\text { para la toma de } \\
\text { decisiones } \\
\text { sobre el proceso } \\
\text { de trabajo }\end{array}$ & $\begin{array}{l}\text { 2. e. } \\
\text { Generación de } \\
\text { áreas o } \\
\text { comisiones de } \\
\text { trabajo }\end{array}$ \\
\hline PRANA & SI & SI & SI & SI & SI \\
\hline PRONOAR & SI & SI & NO & SI & SI \\
\hline $\begin{array}{l}\text { Movimiento } \\
\text { Cajonardi }\end{array}$ & SI & SI & SI & SI & SI \\
\hline Pichangú & SI & NO & SI & SI & SI \\
\hline Sattva & SI & SI & SI & SI & SI \\
\hline Inlackech & NO & NO & SI & SI & SI \\
\hline $\begin{array}{l}\text { La Nueva } \\
\text { Avan* }\end{array}$ & NO & NO & SI & SI & SI \\
\hline La Unión* & SI & $S / D$ & $S / D$ & SI & SI \\
\hline Textil Pigué* & SI & SI & $S / D$ & SI & SI \\
\hline
\end{tabular}

En cuanto a la primera variable de esta categoría, podemos observar que la mayoría de las cooperativas ha realizado modificaciones en las líneas de producción. Por ejemplo, establecer el número justo de trabajadores necesarios en la cadena productiva de 
De Prácticas y discursos/ Universidad Nacional del Nordeste/ Centro de Estudios Sociales

acuerdo al espacio y máquinas disponibles, y los tiempos requeridos entre un paso y otro del proceso; eliminar ingredientes innecesarios para la elaboración de alimentos, cuestión que simplifica el proceso de trabajo y mejora los tiempos de producción; cambios en la ubicación de las máquinas para acortar las distancias de desplazamiento de los trabajadores, modos de señalizar las mercaderías en los estantes para una mejor visualización, entre otras.

En cuanto a la movilidad de los trabajadores entre diferentes puestos y la concentración de tareas de concepción y ejecución -incluidos en la variable 2.a.--, en la mayoría de las cooperativas observamos que hay una preocupación por lograr que los trabajadores conozcan todo el proceso de trabajo y pueda darse un alto grado de rotación entre los puestos. De esta manera, vimos que en la mayor parte de los grupos se intenta encontrar un equilibrio entre un grado de especialización suficiente y necesario en un puesto o rol para desarrollar las tareas con cierta agilidad, y un sistema de rotación en los puestos para evitar la alienación y el aburrimiento. En palabras de los integrantes de las cooperativas:

Aprendimos que estaba bien rotar en función de que por ahí había uno que se saturaba de una tarea o no da más en una tarea y bueno, vamos rotando en algunas cosas, pero generalmente tratamos de mantenerlo el mayor tiempo posible como para que se perfeccione ese compañero en el puesto, encontrarle la vuelta para mejorar la tarea. (Trabajador de Sattva, 16 de mayo de 2015).

Es muy presente cuando alguien entra al grupo, ya de entrada te mostramos todas las partes; la primera vez que vos entres acá, vas a venir a ver todo lo que estamos haciendo y vas a tratar de hacer lo máximo para eso, para que no se produzca ninguna alienación y no se haga nadie indispensable, todos probamos todas las actividades al máximo, y siempre las vamos rotando, si hace tres meses que estoy haciendo la misma tarea, bueno, cambiemos con alguien, así todos estamos en esa cancha, porque en el trabajo manual hay que adquirir una agilidad que te la da el hecho de hacerlo muchas veces. (Trabajadora de PRANA, 28 de agosto de 2015). 
De Prácticas y discursos/ Universidad Nacional del Nordeste/ Centro de Estudios Sociales

Esta rotación permite, por un lado, ampliar por cálculo probabilístico la posibilidad de que surjan innovaciones al pasar más integrantes por el mismo puesto de trabajo y, por otro, facilitar el surgimiento de la innovación al romper con la naturalización de los procesos de producción al presentarle una tarea a alguien no acostumbrado, que por la sola razón de hacer algo nuevo se cuestiona si esa es la mejor forma de hacerlo y busca explicaciones para encontrar la lógica o los criterios ocultos atrás del método que se le presenta como dado.

Esto, sumado a una actitud y conciencia de que se cumple en el grupo un rol activo para proponer cambios y tomar decisiones, genera las condiciones para que puedan darse innovaciones en el proceso de trabajo.

Sin embargo, hemos podido observar también que cuanto mayor es el número de trabajadores en las cooperativas, la posibilidad de rotar los puestos de trabajo se hace más difícil. Esto se da específicamente en las tres cooperativas recuperadas en las cuales, si bien como una postura ideológica los trabajadores estaban de acuerdo en que era necesario rotar "para que todos sepamos hacer todo", luego, en la práctica cotidiana, la mayoría de los trabajadores ocupaba puestos fijos. Incluso, en una de las recuperadas, estaban claramente separadas las tareas de administración y las de ejecución, al punto de que quienes formaban parte de la Comisión Directiva se ocupaban de la administración y no operaban ninguna máquina.

Podemos ver que en varias cooperativas se han creado sistemas informáticos para gestionar la producción o las ventas (variable 2.b). Sólo desarrollaremos a continuación uno de esos ejemplos ya que consideramos que el mismo contiene atributos específicos de la forma en que se concibe el trabajo en estas cooperativas y en que se toman recursos de múltiples lugares para inventar o crear dispositivos. Uno de los almacenes desarrolló una planilla para calcular precios diferenciales para cada cliente, construyendo algunos criterios específicos para hacer descuentos -si el cliente pertenece o no a una cooperativa, volumen y frecuencia de compras anteriores, si el cliente recomendó o no a otros clientes nuevos, si es vecino del barrio o familiar, entre otros-. Lo curioso es la forma en que el trabajador relata haber aprendido a hacer "las fórmulas" para los descuentos: 
En un mundial de fútbol me mandaron un fixture y me llamó la atención que vos ponías los resultados y te hacía cuentas solas; vos ponías el resultado y te ponía tantos puntos, si ponías un empate te sumaba uno, te sumaba tres, entonces me metí en la fórmula, ahí como que empecé a desbloquear eso que me habían mandado, era como ese fixture que a veces llenás a mano, pero este como que ibas agregando y te iba poniendo las posiciones, se cambiaban los equipos iy esto cómo se hace? Y ahí empecé en el país de las fórmulas (Trabajador de PRONOAR, 25 de septiembre de 2015).

A su vez, podemos observar que la mayoría de las cooperativas desarrolla tecnologías de registro (variable 2.c), no sólo de lo que venden y producen, sino también, por ejemplo, de fórmulas -para hacer distintos tipos de cervezas, para producir distintos tipos de piezas de autopartes, para diferentes tratamientos sobre las prendas o, como mencionamos más arriba, de diferentes tipos de "desarrollos tecnológicos"-. También se registran las decisiones tomadas en las reuniones o asambleas que realizan las cooperativas. Estos registros continuos constituyen tecnologías de producción y de gestión que se van aprendiendo en cada grupo y se socializan entre los miembros. En la perspectiva de que suele ser importante para todos los miembros comprender todo el proceso de producción, identificamos que el registro y su socialización constituyen un aprendizaje significativo.

Al mismo tiempo, todos los grupos se organizan a través de comisiones o áreas de trabajo (variable 2.d), por ejemplo, producción, comunicación, administración, logística, vínculos con otras cooperativas, subsidios. En general, la participación en cada una de esas comisiones es voluntaria y ocupada por aquellos que tienen mayores saberes para desarrollar las tareas específicas que requiere cada área o comisión.

Todas las cooperativas realizan de forma frecuente reuniones, más allá de las asambleas anuales formales exigidas por los organismos oficiales. Varias cooperativas tienen identificados incluso distintos tipos de reuniones para trabajar distintos temas. De esta manera, por ejemplo, una de las cooperativas tiene las reuniones "operativas" que son semanales y duran dos horas en las que se resuelven cuestiones concretas de 
De Prácticas y discursos/ Universidad Nacional del Nordeste/ Centro de Estudios Sociales

organización del trabajo durante esa semana -turnos, roles laborales, compras necesarias, organización de los repartos, encadenamiento de las etapas del trabajo, etc., las "de luxe" que son mensuales, en las cuales trabajan sobre la proyección de las ventas, la planificación del trabajo, los ingresos, las inversiones y el lanzamiento de nuevos productos a mediano y largo plazo, y las "special", que son también mensuales en las que trabajan aspectos vinculares que afectan las relaciones entre los integrantes del grupo. En esta organización se identificó que las cuestiones vinculares detonaban a veces en las reuniones operativas y esto impedía lograr una planificación de las tareas cotidianas, poniendo en riesgo la sustentabilidad del emprendimiento al poner en riesgo el trabajo concreto, por lo que se decidió separar estas cuestiones en reuniones específicas.

Así, todas estas cooperativas van produciendo aprendizajes ligados a la autogestión y en forma periódica participan o han participado en algún momento de movimientos, encuentros o jornadas que trabajan sobre la temática de la autogestión. Algunas de ellas incluso han recibido asesoramiento o asistencia técnica por parte de equipos de investigación -incluido el nuestro-, universidades u organismos públicos para adquirir conocimientos específicos -lograr una comunicación más democrática entre todos los integrantes de los grupos, formas de repartir el excedente, producir reglamentos, trabajar conflictos generados en los grupos, entre otras-. A su vez, todas ellas, al participar de los encuentros mencionados, se convierten también en asesores de otras cooperativas de trabajo. La circulación del conocimiento vinculado al "cómo hacer, cómo resolver, cómo pensar cooperativamente" es un eje en continua revisión dentro de cada grupo cooperativo que se acompaña a la vez por una preocupación en ponerlo en discusión con otros grupos.

\section{IV: Discusión}

Como mostramos en los resultados, en las cooperativas que desde sus orígenes se han organizado en torno a la autogestión, se observa un alto grado de desarrollo de tecnologías, tanto aquellas ligadas con el equipamiento como tecnologías de 
De Prácticas y discursos/ Universidad Nacional del Nordeste/ Centro de Estudios Sociales

organización y aprendizaje. En cuanto a las empresas recuperadas, podemos observar que hay situaciones diversas. En tanto se trata de cooperativas que tienen entre 30 y 60 trabajadores, pudimos observar que la posibilidad de rotar en los puestos de trabajo es baja comparándolo con lo que sucede en los otros grupos autogestionados -que en su mayoría tienen entre 5 y 10 integrantes-. Por otro lado, en cuanto al desarrollo de tecnologías, pudimos observar que en dos de las recuperadas no se ha incorporado nueva tecnología ni tampoco se han dado procesos de generación de tecnología. En la recuperada restante sí se han comprado, reparado o adaptado máquinas, y también se han generado dispositivos. Es preciso aclarar que esta recuperada no heredó las máquinas de la empresa anterior, de allí la necesidad de comprar equipamiento o generarlo en comparación con las otras dos recuperadas.

Si, de acuerdo a lo planteado por Quijano (2012), la forma específica en que surge una experiencia es una variable a considerar para establecer su carácter de alternativo, podemos interpretar que para analizar el uso y organización de las fuerzas productivas en estas cooperativas, el hecho de distinguir si ese surgimiento tiene que ver con una postura político ideológica a favor de la autogestión o bien si se opta por esta modalidad como una alternativa para no perder el trabajo, es un punto relevante.

En nuestro país contamos con algunos trabajos que afirman que en la mayor parte de las cooperativas de trabajo, empresas recuperadas y emprendimientos productivos autogestionados no hay un cuestionamiento por parte de los trabajadores acerca de la tecnología y los procesos de organización del trabajo implementados (Fajn, 2003; Parente, 2006). Luego de nuestro análisis, podemos sostener que en el caso de los grupos que desde sus inicios se organizan como cooperativa autogestionada, sí se dan procesos de generación y adaptación de tecnologías -productivas, de organización y de aprendizajes- que contemplan las orientaciones de lo que hemos denominado como proyecto de autonomía. En el caso de las recuperadas, pudimos ver que en aquellas cooperativas en que hay un fuerte compromiso político e ideológico con el proyecto, también se observó la implementación y generación de tecnologías que eran acordes a los principios de la autogestión. Es decir, sostenemos que hay un vínculo entre lo que hemos definido como aprendizajes en torno a la autogestión y la autonomía como 
De Prácticas y discursos/ Universidad Nacional del Nordeste/ Centro de Estudios Sociales

proyecto y la generación de tecnologías tanto aplicadas a la producción como de organización. De hecho, en aquellas cooperativas en las que es más difícil observar prácticas ligadas a la autonomía como proyecto -instancias de formación política e ideológica, participación en movimientos y eventos políticos públicos, relación con el entorno barrial o colaboración con causas justas, acciones educativas o culturales, entre otros-, se observa lo planteado por Novaes (2015: 199), quien llega a la conclusión de que en las recuperadas analizadas por él "está habiendo mucho más continuidades en la discontinuidad que lo opuesto".

Al retomar las dimensiones que hemos desprendido del marco teórico, podemos afirmar que el cuestionamiento de la tecnología y la evaluación de los fines hacia los cuales debe servir se encuentran estrechamente ligados con la postura político ideológica de cada uno de los grupos. En aquellos casos en que se observa una orientación hacia lo que hemos definido como proyecto de autonomía, hay una capacidad en los integrantes de cuestionar modos de hacer y de organizar el trabajo y, por lo tanto, de realizar cambios que beneficien al colectivo. Esto se asocia a su vez con una postura ligada a la conciencia de que el proyecto pertenece a un colectivo y que cada miembro se encuentra en condiciones de igualdad para proponer cuestiones que mejoren el proyecto. Vimos también que en los grupos en que se encuentran estas características se realizan cambios en la materialidad de algunos dispositivos para mejorar las condiciones de trabajo. A su vez, las mejoras tecnológicas permiten en varios casos liberar tiempo de trabajo que se utiliza para diversos fines -acortar la jornada, realizar reuniones, participar en eventos políticos públicos, capacitarse, realizar trabajo comunitario, entre otros-. De esta manera se da una especie de retroalimentación entre las modificaciones que se realizan en las fuerzas productivas y la esfera político ideológica de estos grupos. En este sentido, consideramos importante tomar en cuenta que esto mismo puede ser un aprendizaje significativo e importante de socializar con otros grupos, ya que parece haber una relación dialéctica entre la concepción del proyecto y su práctica cotidiana, siempre y cuando existan modos de objetivar la práctica -productiva, social y política-. Así mismo, identificamos que dicha objetivación se produce en al menos tres esferas concurrentes: la de la práctica 
De Prácticas y discursos/ Universidad Nacional del Nordeste/ Centro de Estudios Sociales

cotidiana y su organización, la de los momentos específicos para pensar sobre dicha práctica y vincularla expresamente con el ideario cooperativo -situaciones que identificamos existen dentro de cada grupo y también se generan entre grupos cooperativos- y en acciones que producen estos grupos con relación a su posicionamiento político de cuestionamiento y propuesta de cambios a nivel sociopolítico ampliado -que en algunos de los grupos ocurre cuando por ejemplo se toma la fábrica, en otros grupos cuando participan de movilizaciones vinculadas al sector y en otros grupos cuando participan de situaciones específicas en donde articulan acciones de difusión de los principios del trabajo autogestionado o de reclamo, propuestas o protesta-.

\section{Conclusiones}

Partimos en este trabajo de pensar que las relaciones sociales incidían en las formas de concebir e implementar tecnologías, a la vez que los propios trabajadores, al usar y generar tecnologías, modelaban también esas relaciones sociales, en otras palabras, que contexto social, tecnología y trabajadores generan una sociodinámica. Pudimos observar que, efectivamente, una forma de organización social diferente, no sólo requiere de tecnologías diferentes, sino que esa forma de organización social alternativa, a través de determinados mecanismos específicos -igualdad entre los miembros, posibilidad de tomar decisiones, orientaciones hacia la justicia, dispersión del poder-, es la que posibilita esas otras formas de interactuar e implementar tecnologías.

Concluimos que en las cooperativas analizadas contar con el conocimiento de todo el proceso de trabajo, sumado a la posibilidad de cuestionar lo dado y proponer otras formas de hacer son las prácticas que abren la posibilidad de generar e implementar tecnologías que persiguen fines diferentes a los que asume el desarrollo tecnológico en el sistema capitalista. Esto tiene algunas implicancias para el análisis que debemos dejar apuntadas para futuros trabajos que aborden la cuestión de la tecnología y sus posibilidades de reformulación. Por un lado, lo que tiene que ver con el tamaño de los 
De Prácticas y discursos/ Universidad Nacional del Nordeste/ Centro de Estudios Sociales

grupos, ya que hemos observado que cuanto mayor era la cantidad de integrantes en las cooperativas, más difícil resultaba la posibilidad de rotar en los puestos de trabajo y también, por lo tanto, de conocer todo el proceso productivo.

Por otro lado, y esto es tal vez lo más relevante, observamos que en aquellas cooperativas en las cuales los trabajadores asumían un fuerte compromiso político y cuestionamiento del orden social vigente, también había una preocupación por implementar modos de trabajo que resguarden la salud física y mental de los trabajadores. Estos modos se trasladaban también a las tecnologías que usan o generan estos grupos, de forma que las mismas pudiesen contemplar estos principios, tal como hemos demostrado en el análisis. Es decir, postulamos que hay una línea de conexión entre lo que hemos definido como proyecto de autonomía y la generación e implementación de tecnologías acordes a esas orientaciones políticas. Consideramos que esto es así porque en estos grupos en los cuales se observa ese compromiso político, hay una actitud por parte de los trabajadores como colectivo de ser conscientes de que el proyecto productivo es propio y, por lo tanto, todos asumen un rol activo en la generación de acuerdos, normas, modos de hacer $\mathrm{y}$, por supuesto, tecnologías. Finalmente, en lo que tiene que ver con las condiciones específicas del surgimiento de cada experiencia, pudimos observar que un origen ligado a la creación de un grupo autogestionado a partir de una convicción política construía un escenario diferente en cuanto a la posibilidad de generar tecnologías alternativas, a diferencia de un grupo que opta por la autogestión como opción frente a la pérdida de una fuente de trabajo. Interpretamos también que resulta más difícil modificar formas de hacer y tecnologías heredadas que partir desde el inicio con una orientación hacia la autonomía.

En el plano metodológico, en tanto, hemos observado que muchas veces las acciones de adaptación y generación de tecnologías tienen que ver con procesos que si bien son reflexionados en el colectivo, se dan de forma cotidiana, a través de la práctica y el ensayo y error; consideramos que la aplicación de técnicas como la observación participante, la entrevista en profundidad y, sobre todo, el trabajo colaborativo y prolongado con los grupos son las adecuadas para poder visibilizar esos procesos. 
De Prácticas y discursos/ Universidad Nacional del Nordeste/ Centro de Estudios Sociales

$\mathrm{Si}$, tal como vimos, las cooperativas autogestionadas que se orientan hacia la autonomía se transforman en espacios privilegiados para la generación de tecnologías alternativas, podríamos pensar en políticas públicas que fomenten la creación y sostenimiento de proyectos que asuman esas características para que, al menos desde esos espacios, surjan las iniciativas para lo que podría ser una nueva dirección del curso en el desarrollo tecnológico.

\section{Bibliografía citada}

Adizes, I. y Wolf, N. (1977). Autogestión: La práctica yugoslava: El efecto de la descentralización sobre los sistemas de organización. México: Fondo de Cultura Económica.

Adorno, T. y Horkheimer, M. (1969). Dialéctica del Iluminismo. Buenos Aires: Editorial Sur.

Bijker, W.; Hughes, T. y Pinch, T. (1989). The Social Construction of Technological Systems. London: MIT Press.

Bookchin, M. (1999). La ecología de la libertad. Madrid: Editorial Nossa y Jara.

Carenzo, S. (2014). Lo que (no) cuentan las máquinas: la experiencia sociotécnica como herramienta económica (y política) en una cooperativa de "cartoneros" del gran Buenos Aires. Antípoda. No 18, pp. 109-135.

Carenzo, S. (2015). Más allá del agregado de valor: Cultura material y socio-génesis tecnológica en la Cooperativa Reciclando Sueños. En Suárez, F. y Schamber, P. J. (comps.) Recicloscopio IV. Miradas sobre dinámicas de gestión de residuos y organización de recuperadores. Buenos Aires: Universidad Nacional de General Sarmiento, pp. 115-142.

Castoriadis, C. (1990). Poder, política, autonomía. En Castoriadis, C. (ed.) El mundo fragmentado. Buenos Aires: Editorial Altamira, pp. 69-90.

Castoriadis, C. (2005). La racionalidad del capitalismo. En Castoriadis, C. (ed.) Figuras de lo pensable. Buenos Aires: Fondo de Cultura Económica, pp. 65-92.

Dagnino, R.; Brandão, F. y Novaes, H. (2004). Sobre o marco analítico-conceitual da tecnología social. En Tecnología social. Uma estratégia para o desenvolvimiento. Rio de Janeiro: Fundacão Banco do Brasil.

De Sousa Santos, B. y Rodríguez, C. (2012). Introducción. En Producir para vivir. México: FCE, pp. $15-61$.

Engels, F. (1972). On authority. Marx-Engels Reader. 730-733. Extraído el 12 de abril de 2010 de: http://www.marxists.org/archive/marx/works/1872/10/authority.htm. 
De Prácticas y discursos/ Universidad Nacional del Nordeste/ Centro de Estudios Sociales

Fajn, G. (2003). Fábricas y empresas recuperadas. Protesta social, autogestión y rupturas en la subjetividad. Buenos Aires: Centro Cultural de la Cooperación.

Feenberg, A. (1991). Introducción: el parlamento de las cosas. En Feenberg, A. (ed.) Critical theory of technology. Oxford: University Press.

Fikey, A. L. y Hanrahan, K. (2014). Moving Beyond Neverland. Reflecting Upon the State of the Diverse Economies Research Program and the Study of the Alternative Economic Spaces. ACME: An International E-Journal for Critical Geographies, Vol. 2, №13, pp. 394-403.

Gaiger, L. I. (2002). A Economia Solidária diante do modo de producao capitalista. Disponible en www.ecosol.org.br. Acceso el 10 de maro de 2010.

Gibson-Graham, J. K. (2008). Intervenciones Posestructurales. Revista Colombiana de Antropología, №38, pp. 261-286.

Habermas, J. (1968). Ciencia y técnica como ideología. Madrid: Editorial Tecnos.

Heidegger, M. (2007). La pregunta por la técnica. Barcelona: Ediciones Folio SA.

Heras Monner Sans, A. I. (2009). Procesos de aprendizaje en proyectos de autonomía. Revista IRICE, N 20, pp. 89-101.

Heras Monner Sans, A. I. (2011). Dispositivos de aprendizaje en autogestión: sus relaciones con el proyecto de autonomía. Intersecciones en Comunicación, Vol. 5, pp. 31-64.

Heras Monner Sans, A. I. (2015). Análisis del aprendizaje sobre la autogestión. La cuestión específica de los aportes y la distribución de recursos. Cuadernos de Antropología Social, $\mathrm{N}^{\circ} 41$, pp. $129-148$.

Heras Monner Sans, A. I. y Burin, D. (2013). Experiencias de incidencia en política pública de organizaciones autogestionadas: desde la autonomía como proyecto hacia la democracia como régimen de sentido. X RAM-Reunión de Antropología del Mercosur, Córdoba, Argentina.

Heras Monner Sans, A. I. y Burin, D. (2014). Para que las diferencias no se transformen en desigualdad. Revista IDELCOOP, N²13, pp. 72-109.

Heras, A. I. y Miano, A. (2014). Sociolinguística y etnografía. Análisis de interacciones en la Mesa de grupos Auto-gestionados. La trama de la comunicación, N 18, pp. 251-271.

Heras, A. I.; Miano, A. y Burin, D. (en prensa). Desde la autonomía como proyecto hacia la democracia como régimen de sentido. En Loera, N. y Fernández Álvarez, M. I. (comps.) La producción de prácticas políticas colectivas.

Infield, H. F. (1971). Sociología de la cooperación: Guía para el estudio de la experimentación social cooperativa. Buenos Aires: Intercoop editores. 
De Prácticas y discursos/ Universidad Nacional del Nordeste/ Centro de Estudios Sociales

Latour, B. (2007). Nunca fuimos modernos. Ensayo de antropología simétrica. Buenos Aires: Siglo XXI.

Lee, R. (2006). The Ordinary Economie: tangled up in values and geography. Transactions of the Institute of British Geographers, New Series, Vol. 31, № 4, pp. 413-432.

Marx, C. (1980). Capital y tecnología (Manuscritos inéditos 1861-1863). México: Terra Nova.

Marx, C. (1984). Miseria de la filosofía. Madrid: Aguilar.

Marx, C. (1987). El Capital. Volúmenes I y III. México: Siglo XXI.

Marcuse, H. (2005). El hombre unidimensional. Barcelona: Ariel.

Mesa Colectiva (2013). La autonomía como proyecto: procesos de reflexión deliberada en experiencias de autogestión, Revista Pueblos y Fronteras, Vol. 3, № 15, pp. 56-91.

Miano, A. (2013). Detonando la burocracia. Estrategias desarrolladas por grupos autogestionados en las interacciones establecidas con el Estado. X RAM-Reunión de Antropología del Mercosur, Córdoba, Argentina.

Moles, A. (1978). Sociodinámica de la cultura. Paidós: Buenos Aires.

Mumford, L. (1982). Técnica y Civilización. Madrid: Editorial Alianza.

Noble, D. (1984). Forces of Production. A Social History of Industrial Automation. Oxford: University Press.

Noble, D. (2001). La locura de la automatización. Barcelona: Alikornio.

Novaes, E. (2015). El fetiche de la tecnología. Buenos Aires: Ediciones Continente.

Parente, D. (2006). Algunas precisiones sobre el determinismo tecnológico y la tecnología autónoma. Una lectura sobre la filosofía de Langdon Winner, Redes, N²3, pp. 79-102.

Quijano, A. (2012). ¿Sistemas alternativos de producción? En Quijano, A. Producción para vivir. México: FCE, pp. 369-399.

Singer, P. (1998). Una utopía militante: repensando o socialismo. Río de Janeiro: Vozes.

Thomas, H. (2012). Tecnologías para la inclusión social en América Latina: de las tecnologías apropiadas a los sistemas tecnológicos sociales. En Thomas, H. (ed.) Tecnología, desarrollo y democracia. Nueve estudios sobre dinámicas socio-técnicas de exclusión/inclusión social. Buenos Aires: Ministerio de Ciencia, Tecnología e Innovación Productiva.

Thomas, H.; Fressoli, M. y Lalouf, A. (2008). Introducción. En Thomas H. y Buch, A. (coords.) Actos, actores y artefactos. Sociología de la tecnología. Bernal: Universidad Nacional de Quilmes, pp. 9-17.

Tiriba, L. (1994). A requalificacao do trabalho: desafio das inovacoes tecnológicas sob o controle fabril dos trabalhadores. $16^{\text {a }}$ Reuniao Anual da Anped. Belo Horizonte. 
De Prácticas y discursos/ Universidad Nacional del Nordeste/ Centro de Estudios Sociales

Toffler, A. (1995). La creación de una nueva civilización. La política de la tercera ola. Barcelona: Plaza y Janés.

Winner, L. (1986). Do artifacts have politics? En Winner, L. The whole and the reactor: a search for limits in an age of high technology. Chicago: University Press, pp. 19-39.

Winner, L. (1992). La carrera tecnológica y la cultura política. En San Martín, J.; Cutcliffe, S. H.; Goldman, S. L. y Medina, M. (eds.) Estudios sobre sociedad y tecnología. Barcelona: Editorial Anthropos, pp. 287-301.

Winner, L. (1993). Upon opening the Black box and finding it empty: Social constructuvism and the Philosophy of Technology. Science, Technology and Human Values, Vol. 18, № 3, pp. 362378. 